\title{
Improved Gardner Suppression Timing Jitter Synchronization Algorithm
}

\author{
Huang Zhen *, Yang Pan, Zhang Wei Han \\ National Institute of Defense Technology, Southwest University of Science and Technology, Mianyang, China \\ Email address: \\ hz1354518117@163.com (Huang zhen),2272117593@qq.com (Yang pan), zhangweihan@163.com (Zhang Wei Han) \\ ${ }^{*}$ Corresponding author
}

\section{To cite this article:}

Huang Zhen, Yang Pan, Zhang Wei Han. Improved Gardner Suppression Timing Jitter Synchronization Algorithm. Journal of Electrical and Electronic Engineering. Vol. 8, No. 1, 2020, pp. 21-26. doi: 10.11648/j.jeee.20200801.14

Received: January 6, 2020; Accepted: February 13, 2020; Published: February 25, 2020

\begin{abstract}
In non-cooperative digital communication systems, the symbol synchronization of the received signal is of great significance for the correct demodulation of the signal. Synchronization, as a key factor in the accurate transmission of information at the sender in communication, is also a vital technology in blind demodulation structures. Strictly speaking, the synchronization technology in non-cooperative communication systems includes not only carrier synchronization and bit synchronization, but also group synchronization and network synchronization. Although the existing synchronization algorithms have good reference value, they also have their limitations. For the traditional symbol synchronization loop based on the Gardner algorithm, when the roll-off coefficient is small and the symbol rate is high, the loop self-noise is serious. This paper uses a symbol synchronization method based on interpolation adjustment sequences, and proposes an improved algorithm that sets a pre-filter that meets the condition of zero timing jitter before the traditional timing error detector to compensate for the effect of self-noise on synchronization. The simulation results of the two algorithms are compared and analyzed. Through the comparison and analysis of the simulation results of the two algorithms, it is found that the improved algorithm can effectively suppress the timing jitter caused by the self-noise of the loop, and at the same time, can accelerate the symbol synchronization speed, effectively improve the limitations of the traditional algorithm and the slow convergence speed. Engineering significance.
\end{abstract}

Keywords: Timing Synchronization, Gardner Algorithm, Filter, Timing Error Detector

\section{Introduction}

Synchronization, as a key factor for accurate transmission of information at the sender side in communication, is also a vital technology in blind demodulation structures. In a strict sense, the synchronization technology in non-cooperative communication systems includes not only carrier synchronization and bit synchronization technology It also includes group synchronization and network synchronization. [1] Although the existing synchronization algorithms have good reference values, they also have their limitations Gardner algorithm based on interpolation to achieve bit synchronization is only applicable to PSK signals; Schmid algorithm can only play its timing synchronization role only in OFDM systems. [2-4] Therefore, the appropriate timing synchronization technology is selected under different modulation modes and channel conditions. It is an important task to be solved in current non-cooperative communication blind receiver design.

Symbol synchronization, also known as bit synchronization, is used to achieve the synchronization between the start and end of each symbol on the blind demodulation side with the local clock. [5] Call up the basis of communication content. At present, there are two main methods to achieve symbol synchronization: the first is to blindly estimate the signal phase parameters and construct a timing synchronization algorithm [6-10]; the other is to perform interpolation processing on the input signal and adjust Input sequence.

This paper uses the second method of symbol synchronization based on interpolation adjustment sequences, and proposes an improved algorithm that sets a pre-filter that meets the condition of zero timing jitter before the traditional timing error detector, and performs simulation results of the two algorithms. Comparative analysis. 


\section{Gardner Algorithm Realizes the Principle of Symbol Synchronization}

The Gardner algorithm achieves symbol synchronization by using an interpolation filter in the timing recovery loop to perform a difference on the resampling input sequence $\mathrm{x}(m T s)$ and output the sequence y $(k T i)$ after interpolation timing [7]. Specifically The block diagram of the symbol synchronization loop is shown in Figure 1:

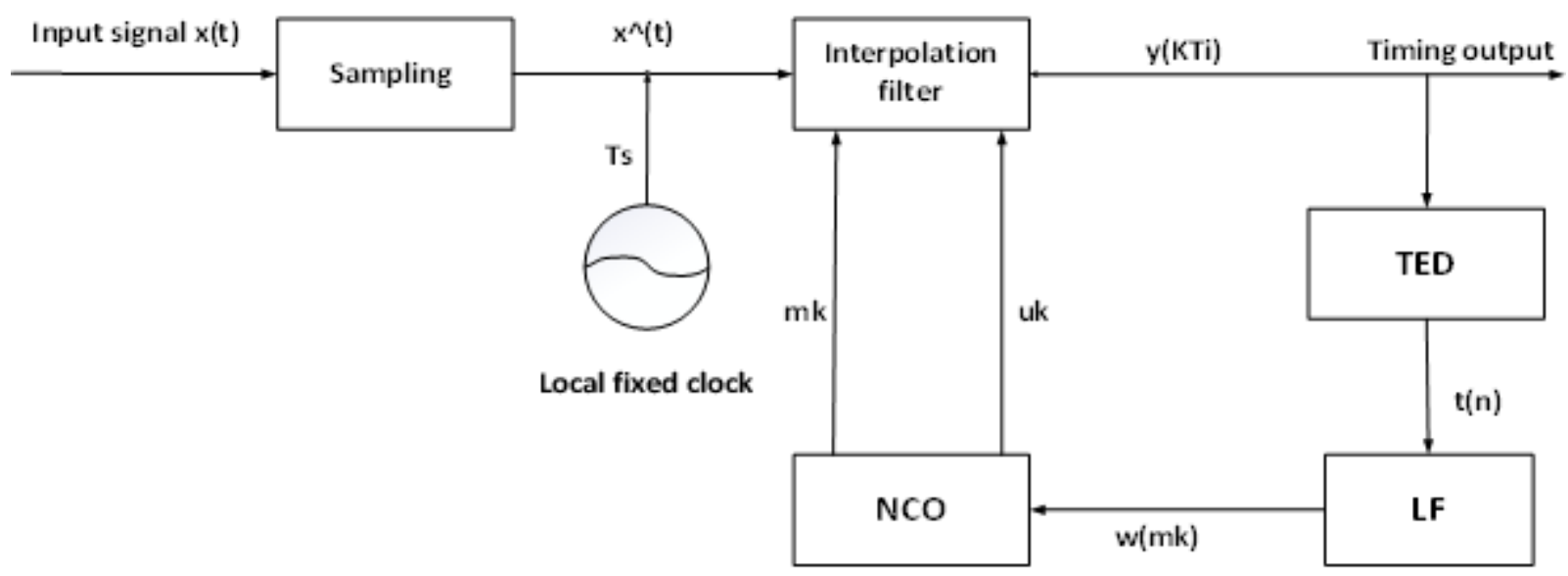

Figure 1. Block diagram of symbol synchronization loop based on Gardner algorithm.

The blind demodulator first rough-demodulates a signal with a period $\mathrm{T}$ to obtain a quasi-baseband signal $x(t)$, and it becomes a discrete sequence $x\left(m T_{S}\right)$ ( $\mathrm{T}$ after a fixed clock sample with a local period Ts and T satisfy the Nyquist basic sampling law, and generally use four times the sampling, that is, Ts $=1 / 4 \mathrm{~T}$. The discrete sequence $x\left(m T_{s}\right)$ is interpolated by the interpolation filter and generated for the symbol Interpolation value of the judgment [8]. If the interpolation value is synchronized, it will be directly output as timing; if the interpolation value is processed by the interpolation filter, it will enter the timing error detector (TED, Timing Error Detector), and the interpolation value and The phase comparison of the local clock obtains the phase error $t(n)$. The phase error signal $t(n)$ includes noise and high frequency components that affect the decision, so $t(n)$ is passed through a loop filter ( $L F$, Loop Filter) To eliminate interference from other ingredients.

In addition, the loop filter outputs the NCO control word $w$ $(m k)$ and sends the control word to a numerically controlled oscillator (NCO, Numerically Controlled Oscillator) [9]. The numerically controlled oscillator calculates the interpolation filter in real time based on the control word $w(m k)$ The integer multiple sampling time $m k$ and the fractional sampling time $u k$, and send the calculation result to the interpolation filter. The interpolation filter interpolates the input sequence according to $m k$ and $u k$, and outputs the synchronized sequence $y(k T i)$.

\section{Improved Gardner Synchronization Algorithm for Timing Jitter Suppression}

The timing recovery loop based on the Gardner algorithm for symbol synchronization has the advantages of simple principle, synchronization performance independent of the carrier phase, and easy implementation [11]. However, this method has the advantages of combating serious timing jitter and slow convergence speed caused by loop self-noise. Obvious deficiency.

(1) The timing error calculated by the Gardner algorithm is estimated based on whether there is a polarity change between the two symbols. When the symbol rate of the communication system is large, the timing error is continued to be calculated in the above manner, and the amplitude of the symbol sequence in the loop is high. Frequency hopping, the self-noise generated by the loop is large, and the timing synchronization performance is seriously affected.

(2) The Gardner algorithm considers that the accurate timing mark is a zero crossing between two symbols and the sample value is zero at the middle of the two best sampling points. ${ }^{[1]]}$ This so-called accurate timing mark must be based on the input signal:

modulus value does not have It is correct only when it is diverse, that is, the signal modulation type can only be MPSK [12]. For non-constant mode signals such as MQAM signals, zero crossing may not occur even between two symbols with the same sign, so the traditional Gardner algorithm is not suitable for multiple Mode signal synchronization.

In addition, the jitter of the timing error signal is also related to the roll-off coefficient of the matched filter in the demodulator, and they have a relatively obvious negative correlation.

In order to achieve the timing synchronization of high-frequency symbols under the small roll-off coefficient of the matched filter and suppress the impact of timing jitter on the symbol synchronization, this paper proposes an improved Gardner timing recovery algorithm.

The improved algorithm adds a pre-filter on the basis of 
the traditional algorithm to pre-filter the signal entering the timing error device. [13-14] The variance of the timing error is only related to the roll-off coefficient of the shaping filter. Within the bandwidth of $(1-a) / 2 T \sim(1+a) / 2 T$ is the effective content of the signal, and the remaining frequency band components act as self-noise. Design a bandpass pre-filter with a band limit of $(1-\alpha) / 2 T \sim(1+a) / 2 T$, Partial filtering of the noise band will effectively reduce timing jitter.

The timing recovery loop under the improved algorithm is shown in Figure 2;

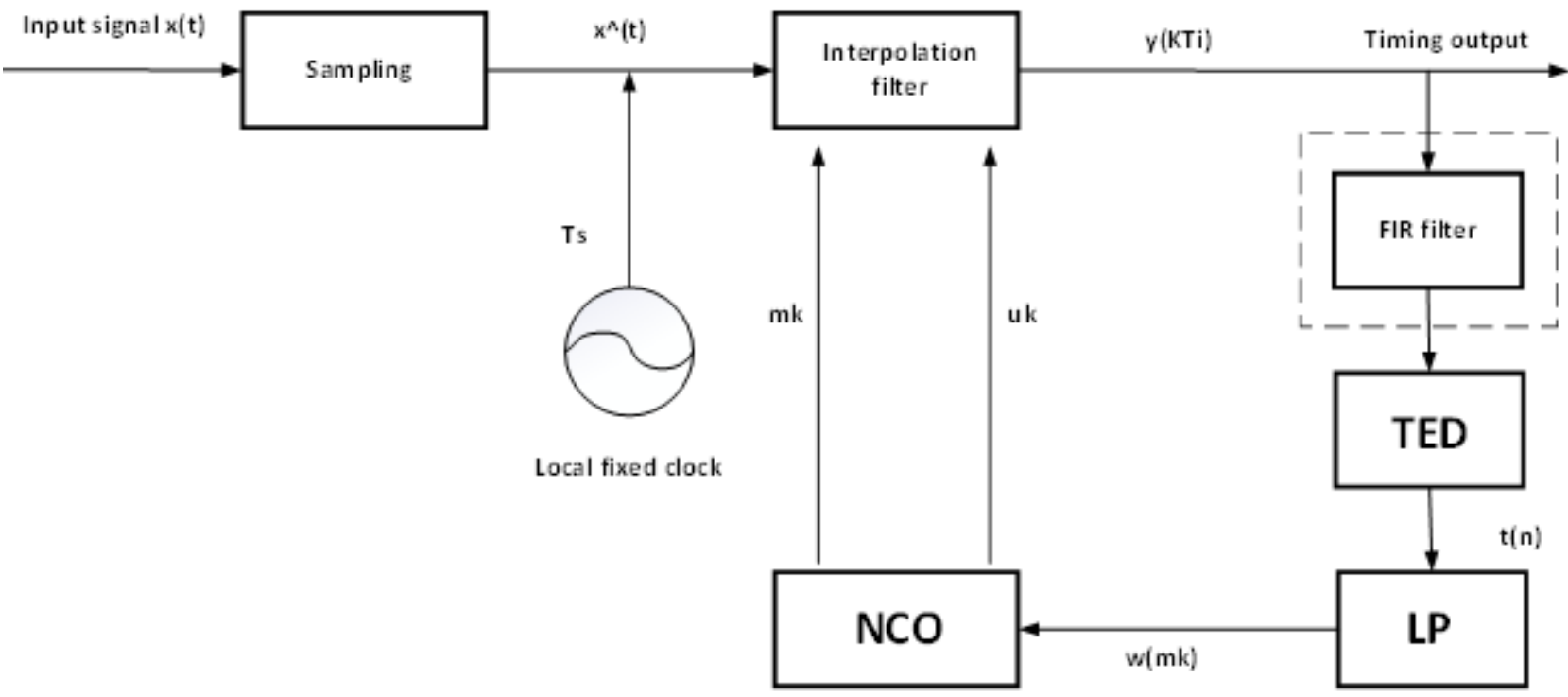

Figure 2. Improved block diagram of the timing recovery loop under the Gardner algorithm.

Let the quasi-baseband sampling signal $x^{\wedge}(t)$ have the characteristics of zero-crossing period with $\mathrm{T}$ as the minimum positive period. After pre-filtering the signal for any $\mathrm{k}$, when $t=k T+T / 2$ is satisfied, there is $x^{\wedge}(t)=0$. Let the pre-filtering function be $f(t)$, the base-band shaping filter function at the transmitting end be $s(t)$, and the coarse-demodulation matching filtering function at the receiving end be $m(t)$. Without considering the loop filter, then The three filters in the entire communication system can be equivalent to:

$$
p(t)=f(t) * m(t) * l(t)
$$

Similarly, for any $\mathrm{k}$, the equivalent filter $\mathrm{p}(\mathrm{t})$ should also satisfy:

$$
p(t)=0, t=K T+T / 2
$$

Let $P(f)$ be the Fourier transform of $p(t)$, then equation (3) can be expressed as:

$$
\sum_{m}^{\infty}(-1)^{m} p(f-m / T)=0
$$

Further, assuming that $\mathrm{P}$ (f) has a periodic spectrum characteristic with a bandwidth less than $1 / \mathrm{T}$, equation (4) can be further expressed as:

$$
p(f)=p(f-1 / T), 0 \leq f<1 / T
$$

Let $M(f)$ be the spectral expression of the raised cosine signal obtained by baseband signal shaping filtering. At this time, the Fourier transform of the pre-filter function that can suppress timing jitter can be expressed as:

$$
P(f)= \begin{cases}1 / T \cdot M(f-1 / T), & 0 \leq f<1 / T \\ 1 / T \cdot M(f+1 / T), & -1 / T \leq f<0\end{cases}
$$

Perform the inverse Fourier transform on the above formula to get the pre-filter function:

$$
f(t)=m(t) \cos (\pi t / T)
$$

\section{Simulation Analysis}

\subsection{Matalab Simulation and Analysis of Traditional Gardner Algorithm with Large Roll-off Coefficient}

The simulation environment is MATLAB R2015, the signal modulation method is BPSK, and the raised-cosine [13] shaped roll-off filter is used to shape and filter the baseband signal. The basic parameters of the modulation end are set as follows: symbol length 1000, shaping filter coefficient $a=$ 0.7 , symbol rate 1000 , sampling frequency $1200 \mathrm{KHz}$. The channel uses a Gaussian channel with a noise intensity of $20 \mathrm{dBW}$. The basic parameters of the blind demodulator are set as follows: the phase deviation is set to 0 , the matched filter order is 64 , and quadruple resampling is used to periodically recover the loop gain The coefficient $C 1=2 * 10^{\wedge}(-3)$, the initial value of the NCO control word $w$ $(m k)$ is set to 0.5 , the initial value of the $\mathrm{NCO}$ register is 0.6 , and the initial value of the fractional interpolation coefficient is set to 0.6 .

The relationship between the nco control word and the number of iterations is expressed by: 


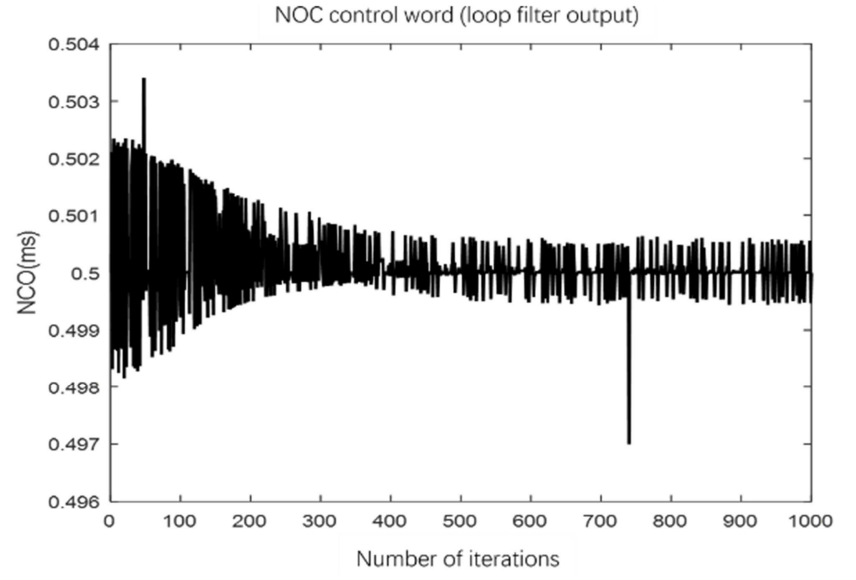

Figure 3. Changes of NCO control words of traditional Gardner algorithm under large roll-off coefficient.

From Figure 3, it can be seen that when the number of iterations is small, the value of the control word is unstable and the range of change is large. The value of the control word is basically stable between 0.4995 and 0.5005 when the number of iterations is about 400 , and the output of the loop filter converges. Better performance.

Figure 4 represents the change curve of the fractional coefficient $u k$ with the number of iterations. The fractional coefficient $u k$ gradually approaches zero during the iteration process, but the convergence process is relatively slow, and it is basically stable at zero when the number of iterations approaches 1800. Traditional Gardner The algorithm has a clear convergence trend for $u k$ when the matching filter roll-off coefficient is large, but there is a shortcoming in the convergence speed, and it needs a large number of sampling points to obtain better convergence performance.

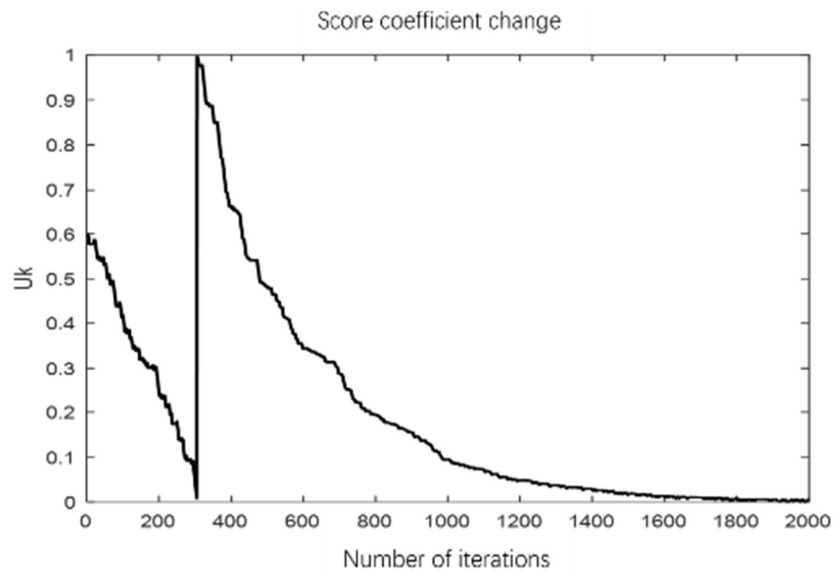

Figure 4. Variation of the fractional spacer value (uk) of the traditional Gardner algorithm under the large roll-off factor.

Synthesizing the convergence curves of the uk and NCO control words, it can be seen that when the demodulator matched filter roll-off coefficient is large, the synchronization effect of the symbol synchronization timing loop under the traditional Gardner algorithm on the BPSK signal is obvious, but the synchronization speed is slow and the synchronization is reached. The required number of sampling points is large. If the demodulator is not too demanding, the traditional Gardner algorithm can be used as a symbol synchronization algorithm for constant-mode signals.

\subsection{Analysis and Comparison of Matalab Simulation Results of two Algorithms with Small Roll-off Coefficient}

The experimental simulation environment remains unchanged, changing the roll-off coefficient of the matched filter to $a=0.3$, increasing the symbol rate at the sending end to $5 * 106$, and the sampling frequency is 8 times the symbol rate. Increasing the symbol rate and sampling frequency can easily make the symbols The sequence generates timing jitter during the transition, which is more conducive to observing the simulation results.

First, compare the change curves of the NCO control word $(w(m k))$ with the increase of the number of iterations under the two algorithms:

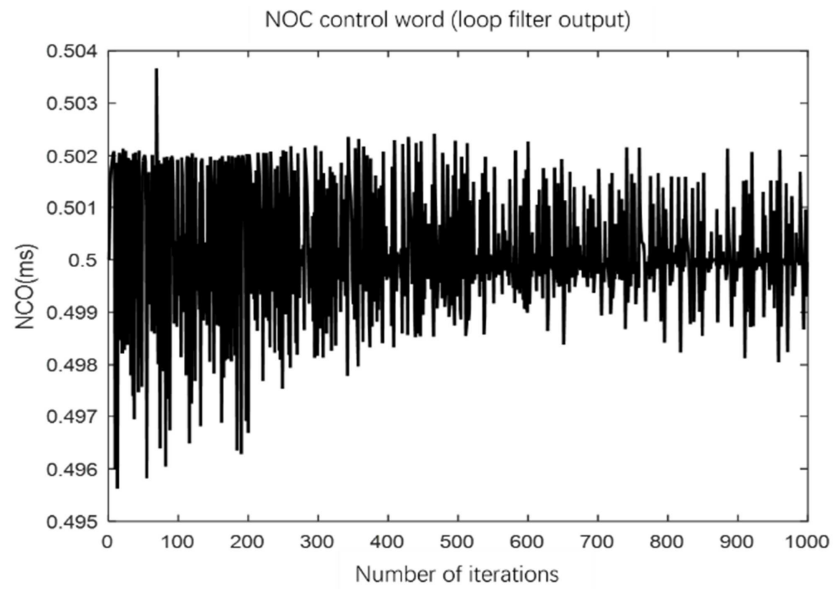

Figure 5. Change of NCO control word of traditional Gardner algorithm under small roll-off coefficient.

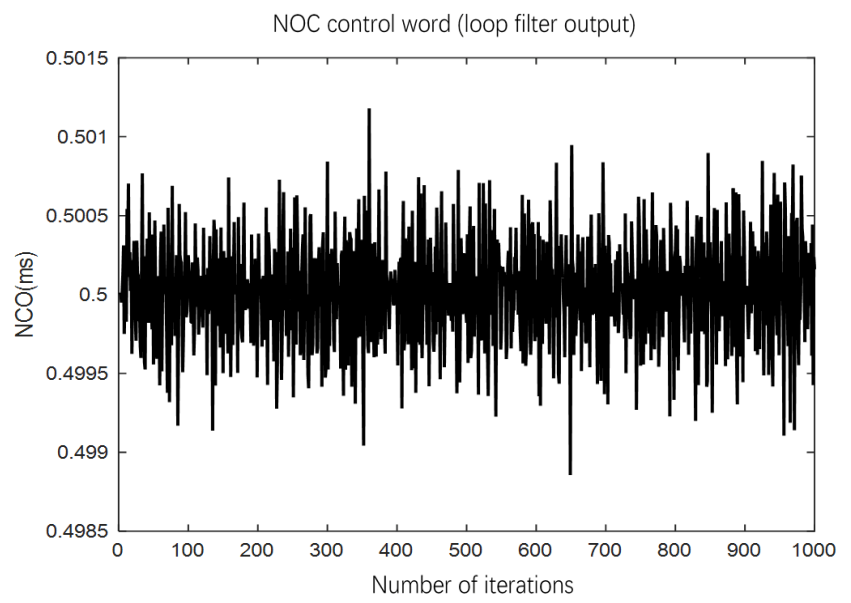

Figure 6. Improvement of Gardner algorithm NCO control word change under small roll-off factor.

When the matched filter roll-off coefficient is small, the traditional timing recovery loop's nco control word converges poorly. Even after the number of iterations is over half, the control word still beats continuously in the range of 
approximately $0.496-0.502$; and the improved algorithm's nco control The word has only changed in the range of $0.499-0.501$ from the beginning. It can be seen that the improved algorithm has better stability for the nco control word and effectively solves the problem of slow convergence speed.

Next, observe the comparison chart of the change of the score coefficient $u k$ under the traditional Gardner algorithm and the improved algorithm:

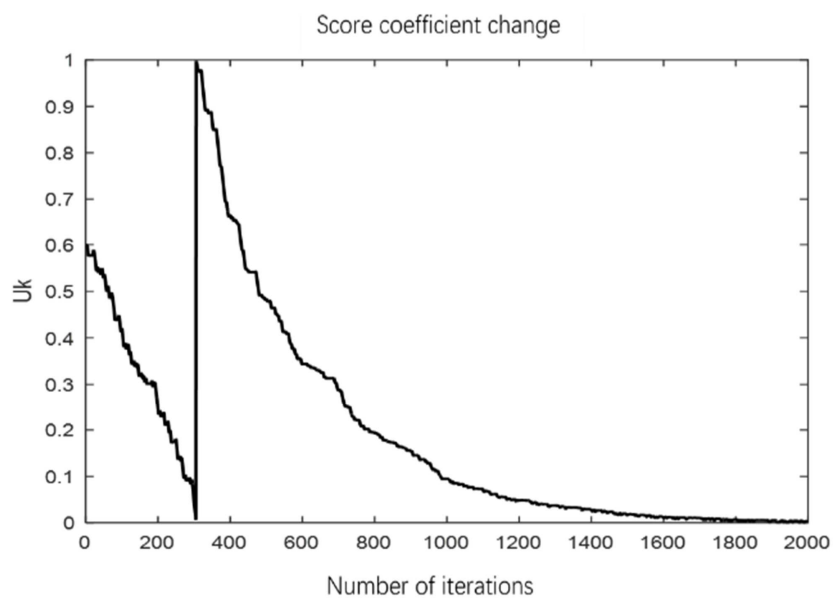

Figure 7. Variation of the fractional spacer value $(u k)$ of the traditional Gardner algorithm under the small roll-off factor.

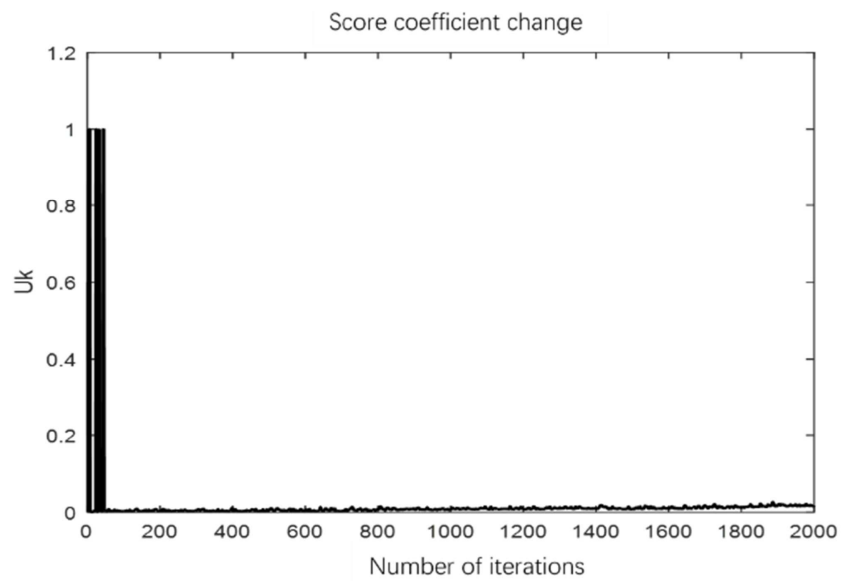

Figure 8. Variation of the fractional spacer value (uk) of the improved algorithm under the small roll-off factor

Figures 7 and 8 show the convergence curves of uk under the traditional Gardner algorithm and the improved algorithm when the matched filter roll-off coefficient is small. In the traditional Gardner algorithm, $u k$ converges slowly, and does not converge even when the number of iterations reaches 2000 To zero; the improved algorithm $u k$ convergence curve, when the number of iterations is less than 100, $u k$ has basically stabilized at zero.

Combining the changes of the NCO control word and the fraction coefficient $u k$ in the two algorithms, the following conclusions can be drawn:

When the symbol rate at the transmitting end is large and the demodulator matched filter roll-off coefficient is small, the symbol synchronization performance of the traditional
Gardner algorithm is poor. On the one hand, the value of the NCO control word output by the loop filter is not good. Stable at about 0.5 , on the other hand, it shows that the fractional coefficient $u k$ converges to zero is too slow. In contrast, the improved algorithm has better synchronization performance, indicating that the method of adding a prefilter plays a role in suppressing the self-noise of the loop. Effect, reducing the impact of loop timing jitter on symbol synchronization.

\section{Conclusion}

The traditional symbol synchronization loop based on the Gardner algorithm has a large matched filter roll-off coefficient in the demodulator, and is suitable for MPSK signals when the symbol rate on the transmitting end is low. When the roll-off coefficient is small and the symbol rate is high, the loop self-noise is severe, and the traditional timing loop cannot achieve the ideal timing synchronization effect. This paper proposes an improved algorithm that sets a pre-filter that satisfies the condition of zero timing jitter in front of the traditional timing error detector to compensate for the effect of self-noise on synchronization. Through the comparison and analysis of the simulation results of the two algorithms, it is found that the improved algorithm can effectively suppress the timing jitter caused by the self-noise of the loop, at the same time, can accelerate the symbol synchronization speed, effectively improve the limitations of the traditional algorithm and the slow convergence speed. The method has certain practical engineering significance.

\section{References}

[1] Liu Wei, Yao Yuanyuan, Qin Mingwei. An improved Gardner timing synchronization algorithm [J]. Computer Engineering, 2013, 39 (11): 299-302.(Liu Wei, Yao Longyuan, Qin Mingwei. An Improved Gardner Timing Synchronization Algorithm [J]. Computer Engineering, 2013, 39 (11): 299-302).

[2] Zhu Xueyang. Research on Bit Timing Synchronization Based on Gardner Algorithm [D]. Nanjing University of Science and Technology, 2010. (Zhu Xueyang. Research on Bit Timing Synchronization Based on Gardner Algorithm [D]. Nanjing University of Science and Technology, 2010).

[3] Fu Yongming, Zhu Jiang, Yunying Ying, Bai Hong. Research on Gardner Timing Synchronization Loop Self-Noise Reduction Algorithm [J]. Modern Electronic Technology, 2011, 34 (21): 92-95.(Fu Yongming, Zhu Jiang, Ju Yingyu, Bai Hong. Study on the self-noise reduction algorithm of Gardner timing synchronization lo op [J]. Modern Electronic Technique, 2011, 34 (21): 92-95).

[4] Liu Yingchao, Qiu Yuanteng, Li Chunhai. Simulation and Implementation of Gardner Bit Timing Algorithm Based on Interpolation [J]. Electronic Technology, 2017, 46 (11): 51-53.(Liu Yingchao, Qiu Yuanteng, Li Chunhai. Simulation and Implementation of Gardner Bit Timing Algorithm Based on Interpolation $[\mathrm{J}]$. Electronic Technology, 2017, 46 (11): 51-53). 
[5] Wang Lei, Sui Qiang, Du Changyin, Liu Hao. Gardner Algorithm and Bit Synchronization Loop Design for Self-Noise Suppression [J]. Infrared and Laser Engineering, 2017, 46 (06): 187-192.(Wang lei, Yu Qiang, Du Changwei, Liu Wei. Design of self-noise suppression Gardner algorithm and bit synchronization loop [J]. Infrared and laser Engineering, 2017, 46 (06): 187-192).

[6] Feng Ran, Han Jun. A Gardner timing error detection algorithm approaching zero jitter [J]. Radio Engineering, 2016, 46 (01): 50-52 + 56. (Feng Yiran, Han Jun. A Gardner Timing Error Detection Algorithm Approaching Zero Jitter [J]. Radio Engineering, 2016, 46 (01): 50-52+56).

[7] Zhao Yanli. Improved method based on Gardner timing recovery algorithm $[\mathrm{A}]$. Sichuan Provincial Communications Society. Proceedings of the Sichuan Provincial Communications Society's 2013 Annual Conference [C]. Sichuan Provincial Communications Society: Sichuan Provincial Communications Society, 2013: 5.(Zhao Yanli. An Improved Method Based on Gardner Timing Recovery Algorithm [A]. Sichuan Communication Society. Sichuan Communication Society 2013 Annual Conference Proceedings [C]. (Sichuan Communication Society: Sichuan Communication Society, 2013: 5).

[8] Gardner Denver to combine with Ingersoll-Rand's Industrial business [J]. Filtration Industry Analyst, 2019, 2019 (5).
[9] Gardner Denver supplies medical vacuum technology [J]. World Pumps, 2019, 2019 (6).

[10] Efficacy and complications of the use of Gardner-Wells Tongs: a systematic review [J]. Journal of Spine Surgery, 2018, 4 (1).

[11] Baldino Maria Eduarda, Koth Valesca Sander, Silva Daniela Nascimento, Figueiredo Maria Antonia, Salum Fernanda Gonçalves, Cherubini Karen. Gardner syndrome with maxillofacial manifestation: A case report. [J]. Special care in dentistry: official publication of the American Association of Hospital Dentists, the Academy of Dentistry for the Handicapped, and the American Society for Geriatric Dentistry, 2019, 39 (1).

[12] Bokaeeyan Mahyar, Ankiewicz A, Akhmediev N. Bright and dark rogue internal waves: The Gardner equation approach. [J]. Physical review. E, 2019, 99 (6-1).

[13] Larsson Wexell Cecilia, Bergenblock Sibel, Kovács Anikó. A Case Report on Gardner Syndrome With Dental Implant Treatment and a Long-Term Follow-Up.[J]. Journal of oral and maxillofacial surgery: official journal of the American Association of Oral and Maxillofacial Surgeons, 2019, 77 (8).

[14] Polynomials; Reports on Polynomials from Eskisehir Osmangazi University Provide New Insights (Numerical solutions of the Gardner equation by extended form of the cubic B-splines)[J]. Journal of Technology \& Science, 2018. 